\title{
Angelica Sinensis promotes myotube hypertrophy through the PI3K/Akt/mTOR pathway
}

\author{
Tzu-Shao Yeh", Cheng-Chen Hsu' ${ }^{2+}$, Suh-Ching Yang ${ }^{1+}$, Mei-Chich Hsu ${ }^{3,4^{*}}$ and Jen-Fang Liu ${ }^{1,5,6^{*}}$
}

\begin{abstract}
Background: Angelica Sinensis (AS), a folk medicine, has long been used in ergogenic aids for athletes, but there is little scientific evidence supporting its effects. We investigated whether AS induces hypertrophy in myotubes through the phosphatidylinositol 3-kinase (PI3K)/Akt (also termed PKB)/mammalian target of the rapamycin (mTOR) pathway.

Methods: An in vitro experiment investigating the induction of hypertrophy in myotubes was conducted. To investigate whether AS promoted the hypertrophy of myotubes, an established in vitro model of myotube hypertrophy with and without AS was used and examined using microscopic images. The role of the PI3K/Akt/mTOR signaling pathway in AS-induced myotube hypertrophy was evaluated. Two inhibitors, wortmannin (an inhibitor of $\mathrm{PI} 3 \mathrm{~K}$ ) and rapamycin (an inhibitor of mTOR), were used.
\end{abstract}

Result: The results revealed that the myotube diameters in the AS-treated group were significantly larger than those in the untreated control group $(P<0.05)$. Wortmannin and rapamycin inhibited AS-induced hypertrophy. Furthermore, AS increased Akt and mTOR phosphorylation through the PI3K pathway and induced myotube hypertrophy.

Conclusion: The results confirmed that AS induces hypertrophy in myotubes through the PI3K/Akt/mTOR pathway.

Keywords: C2C12, Dong Quai, Muscle, IGF-1

\section{Background}

Muscle mass is a primary determinant of muscle strength, and is strongly associated with the performance of activities of daily living and the level of independence of the elderly [1-3]. The phosphatidylinositol 3-kinase (PI3K)/ Akt (also termed PKB)/mammalian target of rapamycin (mTOR) pathway is recognized as a possible mechanism that regulates muscle mass [4-6]. In mammals, skeletal muscle hypertrophy occurs as a result of an increased size, instead of increased number, of preexisting skeletal muscle fibers $[7,8]$. The effects of this pathway on skeletal muscle are exhibited most prominently downstream of insulin-like growth factor 1 (IGF-1) signaling. The prohypertrophic activity of IGF-1 predominantly results from activation of the PI3K/Akt/mTOR signaling pathway [9]. Akt is a serine-threonine protein kinase that can inhibit

\footnotetext{
* Correspondence: meichich@kmu.edu.tw; liujenfa@gw.cgust.edu.tw ${ }^{\dagger}$ Equal contributors

${ }^{3}$ Department of Sports Medicine, Kaohsiung Medical University, Kaohsiung 80708, Taiwan

${ }^{1}$ School of Nutrition and Health Sciences, Taipei Medical University, Taipei 11031, Taiwan

Full list of author information is available at the end of the article
}

the induction of muscle atrophy $\mathrm{F}$ box and muscle RINGfinger protein 1 ubiquitin-ligases by using forkhead transcription factor FOXO1 (also called "forkhead"), resulting in the prevention of muscle atrophy $[10,11]$. Furthermore, activating Akt is sufficient to prevent muscle atrophy [12], and the kinase activity of Akt is essential for IGF-1induced hypertrophy [13]. The aforementioned findings imply that the PI3K/Akt/mTOR pathway plays a pivotal role in muscle hypertrophy and atrophy.

The $\mathrm{C} 2 \mathrm{C} 12$ cell line, a myoblast cell line derived from murine satellite cells, is used extensively as an in vitro model to study both muscle differentiation and hypertrophy [14]. The withdrawal of serum from $\mathrm{C} 2 \mathrm{C} 12$ myoblasts leads them to exit the cell cycle and fuse into myotubes. $\mathrm{C} 2 \mathrm{C} 12$ myotubes have been used in in vitro models to study IGF-1 mediated hypertrophic signaling pathways in skeletal muscle $[9,15,16]$. PI3K/Akt/mTOR activation downstream of IGF-1 can induce hypertrophy both in $\mathrm{C} 2 \mathrm{C} 12$ cells in vitro [13] as well as in skeletal muscle in vivo [12]. Thus, $\mathrm{C} 2 \mathrm{C} 12$ myotubes provide a useful, well-characterized, in vitro modelling system regarding the induction of hypertrophy in myotubes. 
China has a long history of using natural products as ergogenic aids to enhance athletic performance. The dried root of Angelica Sinensis (AS) is widely used in traditional Chinese medicine to "nourish one's vitality and enrich blood," which means increasing the stamina of weak patients and improving their strength. The main chemical constituents of AS roots are ferulic acid, ligustilide, angelicide, brefeldin A, butylidenephthalide, butyphthalide, succinic acid, nicotinic acid, uracil, and adenine [17]. The constituents most often associated with the pharmacological activities of AS roots are ferulic acid and ligustilide (predominantly the $Z$-isomer). Ferulic acid can inhibit platelet aggregation and serotonin release, and ligustilide exhibits significant antiasthmatic and spasmolytic activities [17]. The levels of these 2 constituents are typically used as chemical markers for the quality control of AS roots $[18,19]$. Some of these roots are thought to exhibit proliferous properties, whereas others might exhibit myogenesis effects. Ferulic acid, an active compound derived from AS, can stimulate cell proliferation through Akt signaling [20]. Polysaccharides in AS roots can promote the proliferation and differentiation of hematopoietic stem and progenitor cells and megakaryocytic lineages through the PI3K/Akt pathway [21]. However, whether AS actually induces hypertrophy in myotubes is unknown. Therefore, we investigated whether AS induces hypertrophy in myotubes through the PI3K/Akt/mTOR pathway. An in vitro experiment regarding the induction of hypertrophy in myotubes was conducted.

\section{Methods}

\section{Cell culture}

Mouse skeletal muscle cells, $\mathrm{C} 2 \mathrm{C} 12$ myoblasts, were purchased from the Bioresource Collection and Research Center (Food Industry Research and Development Institute, Hsinchu, Taiwan). Cells were maintained in 90\% Dulbecco's modified Eagle's medium (DMEM; 11965; Gibco, Invitrogen, Carlsbad, CA, USA), supplemented with 10\% fetal bovine serum (10437; Gibco, Invitrogen, Carlsbad, CA, USA) and $1 \%$ penicillin-streptomycin-amphotericin $B$ (P/S/A; Biological industries, Kibbutz, Beit HaEmek, Israel) at $37^{\circ} \mathrm{C}$ in a $5 \% \mathrm{CO}_{2}$ atmosphere. Differentiation was induced by changing the medium to DMEM containing $2 \%$ horse serum (HS) and $1 \% \mathrm{P} / \mathrm{S} / \mathrm{A}$ when the cells attained $90 \%$ confluence $[9,22]$. The myotubes matured to striated cells by the fifth day after sowing, and the cultures were used for experiments as described previously [23]. The myotubes were assigned to 3 groups to investigate the effects of AS on myotubes: (1) non-AS supplement (normal growth medium: 2\%HS/DMEM; NON); (2) IGF-1 supplement (10 ng/mL, in $2 \% \mathrm{HS} / \mathrm{DMEM}$; as a positive control; IGF-1); and (3) AS supplement (10 ng/mL, in 2\% HS/DMEM; AS).

\section{Herbal and chemical reagents}

The herbal and chemical reagent stocks used were as follows: AS $(10 \mathrm{mg} / \mathrm{mL}$; HerBesta, ITS-TW, Taipei, Taiwan), wortmannin (1 $\mu \mathrm{M}$; inhibitor of PI3K, W1628, Sigma, St. Louis, MO, USA), rapamycin (100 ng/mL; inhibitor of mTOR, R0395, Sigma, St. Louis, MO, USA), and IGF-1 (100 ng/mL, I8779, Sigma, St. Louis, MO, USA). The AS and chemicals were dissolved separately in phosphate-buffered saline ( $137 \mathrm{mM} \mathrm{NaCl}, 8.10 \mathrm{mM}$ $\mathrm{Na}_{2} \mathrm{HPO}_{4}, 2.68 \mathrm{mM} \mathrm{KCl}, 1.47 \mathrm{mM} \mathrm{KH} \mathrm{PO}_{4}, \mathrm{pH}$ 7.40). The stocks were stored in aliquots at $-20^{\circ} \mathrm{C}$. Regarding treatment, the stocks were diluted in the medium and added directly to the cultured cells according to the following final concentrations: AS $\left(1,10,10^{2}, 10^{3}, 10^{4}, 10^{5}\right.$, $10^{6} \mathrm{ng} / \mathrm{mL}$ in $2 \% \mathrm{HS} / \mathrm{DMEM}$ ), wortmannin (100 $\mathrm{nM}$ in DMEM), rapamycin (10 ng/mL in DMEM), and IGF-1 (10 ng/mL in 2\% HS/DMEM; as a positive control for the activated PI3K/Akt/mTOR pathway).

\section{Assessment of Angelica Sinensis cytotoxicity in myotubes through an XTT assay}

C2C12 cells were cultivated in a flat 96-well plate at a density of $5 \times 10^{3}$ cells per well, and incubated for $5 \mathrm{~d}$ to permit the maturation of the myotubes into striated cells. AS was added to the myotubes at various concentrations $\left(1,10,10^{2}, 10^{3}, 10^{4}, 10^{5}, 10^{6} \mathrm{ng} / \mathrm{mL}\right)$ after the myotubes matured. After 24, 48, and $72 \mathrm{~h}$, an XTT (2, 3Bis (2-methoxy-4-nitro-5-sulfophenyl)-2H-tetrazolium5-carboxanilide inner salt) reagent (Biological industries, Kibbutz, Beit HaEmek, Israel) was added to each well according to the manufacturer's instructions. After $2 \mathrm{~h}$ in the culture, cell viability was determined by measuring the absorbance at $490 \mathrm{~nm}$, using a 550 BioRad plate reader (Bio-Rad, Hertfordshire, UK). Dose and time course experiments were performed in quadruplicate.

\section{Myotube hypertrophy based on measurement of myotube diameter}

The $\mathrm{C} 2 \mathrm{C} 12$ cells were seeded at a density of $2 \times 10^{5}$ cells in 6-well plates (BD Biosciences, Sparks, MD, USA). The myotubes were matured after $5 \mathrm{~d}$, and used in the experiments. To conduct the AS-induced myotube hypertrophy experiment, the myotubes were treated with AS (10 ng/mL, AS in 2\% HS/DMEM) or fresh growth medium (DMEM containing 2\% HS; NON) and incubated for $72 \mathrm{~h}$; the myotube diameters were then determined. To determine the effects of inhibitors on AS-induced hypertrophy, the myotubes were treated with or without inhibitors (wortmannin or rapamycin) $30 \mathrm{~min}$ before the trials. The culture medium was replaced with IGF-1 (10 ng/mL, in 2\% HS/DMEM), AS (10 ng/mL, in $2 \% \mathrm{HS} /$ DMEM), or fresh growth medium (2\% HS/DMEM; $\mathrm{NON})$. The trials were conducted at $37^{\circ} \mathrm{C}$ in an atmosphere of $5 \% \mathrm{CO}_{2}$. After $72 \mathrm{~h}$ of incubation, the myotube 
diameters were examined. All experiments were performed in triplicate.

The myotube diameters were determined using a light microscope (Olympus CKX41, with a 20× objective lens; Olympus, Tokyo, Japan) with a digital camera system (Olympus C7070; Olympus, Tokyo, Japan) and MediaCybernetic Image-Pro Plus software (MediaCybernetic, Bethesda, MD, USA). Each group was cultured in 3 wells, and each well was evenly divided into 9 square grid sections. Three images for each section were captured. At least 10 myotubes per image were measured. Three short-axis measurements were taken along the length of a given myotube diameter and the average was calculated.

\section{Western blotting}

The myotubes were treated with AS $(10 \mathrm{ng} / \mathrm{mL})$ at various time points, and the time point that exhibited the highest protein expression of phosphospecific Akt and mTOR was identified using western blotting. According to the time point that exhibited the highest level phosphospecific of Akt and mTOR, the myotubes were treated with AS, and $1 \mu \mathrm{M}$ wortmannin, an inhibitor of PI3K, was added for $30 \mathrm{~min}$ to break the PI3K/Akt/ mTOR pathway. After incubation, the myotubes from the cell culture plate were scraped into an eppendorf tube to analyze the protein levels of phosphorylated Akt on $\operatorname{Ser}^{473}$ (p-Akt) and mTOR on $\operatorname{Ser}^{2448}$ (p-mTOR) (Cell Signaling Technology, Beverly, MA, USA). This analysis was conducted using western blotting.

Cells were lysed using a CelLytic Extraction Kit (SigmaAldrich, St. Louis, MO, USA) with $1 \%$ phosphatase inhibitor cocktail 3 (Sigma-Aldrich, St. Louis, MO, USA). Quantification was performed using a protein assay (Bio-Rad Laboratories, Hercules, CA, USA). Samples containing $50 \mu \mathrm{g}$ of total protein were separated using sodium dodecyl sulfate polyacrylamide gel electrophoresis for $150 \mathrm{~min}$ at $120 \mathrm{~V}$ by applying $8 \%$ gradient gels on a Criterion electrophoresis cell (Bio-Rad Laboratories, Richmond, CA, USA).

Proteins were transferred to a polyvinylidene fluoride membrane (PALL Gelman Laboratory, Taipei, Taiwan) at a $100-\mathrm{mA}$ constant current for $10 \mathrm{~h}$ on ice at $4^{\circ} \mathrm{C}$. The membrane was blocked in a tris-buffered saline (TBS) solution containing $0.1 \%$ Tween 20 (TBS-T) and 5\% nonfat dry milk for $1 \mathrm{~h}$ and then incubated overnight at $4^{\circ} \mathrm{C}$, using commercially available rabbit polyclonal primary phosphospecific antibodies. These antibodies recognized the phosphorylated Akt on $\mathrm{Ser}^{473}$, mTOR on $\mathrm{Ser}^{2448}$ (Cell Signaling Technology, Beverly, MA, USA), and $\beta$-actin (Sigma-Aldrich, St. Louis, MO, USA).

All antibodies were diluted to a 1:500 ratio in TBS-T containing $5 \%$ nonfat dry milk (except $\beta$-actin, which was diluted to a ratio of 1:10 000). The membranes were then washed in TBS-T, incubated using a secondary antibody (horseradish peroxidase-conjugated antirabbit IgG; Sigma-Aldrich, St. Louis, MO, USA), and diluted to a ratio of 1:16 000 in TBS-T with $5 \%$ milk for $1 \mathrm{~h}$, followed by washing in TBS-T. Phosphorylated proteins were visualized using enhanced chemiluminescence according to the manufacturer's protocols (Pierce Biotechnology, Rockford, IL, USA) and quantified using MediaCybernetic Image-Pro Plus software (MediaCybernetic, Bethesda, MD, USA).

The membranes described above were incubated in Restore Western Blot Stripping Buffer (Pierce Biotechnology, Rockford, IL, USA) for $30 \mathrm{~min}$ and reprobed using the appropriate antibodies for detecting the total expression levels of Akt, mTOR (rabbit monoclonal primary antibody) (Cell Signaling Technology, Beverly, MA, USA), and $\beta$-actin by using western blot analysis. All experiments were performed in triplicate.

\section{Statistical analysis}

All values were expressed as the mean \pm standard deviation (SD). The myotube diameters of the 2 treatments (AS vs. NON) were compared using a Student's $t$ test. The phosphorylation levels of Akt or mTOR at various treatment time points were analyzed using a one-way analysis of variance (ANOVA). Group and treatment effect data were analyzed using a 2-way ANOVA combined with Scheffe posthoc analysis. Significance was determined at the $P<0.05$ level. All tests were performed using Statistical Package for Social Science (SPSS, Chicago, IL, USA) software Version 14.0 for Microsoft Windows.

\section{Detection of ferulic acid in Angelica Sinensis by using high performance liquid chromatography}

To confirm the quality of the AS, we detected its main chemical constituents. The amount of ferulic acid in the AS was analyzed using a high performance liquid chromatographic method [24]. Quantification was accomplished using a comparison of the peak areas of the sample with those of the reference standard. The amount of ferulic acid in the AS was $0.61 \mathrm{mg} / \mathrm{g}$ (Additional file 1: Figure S1 and S2).

\section{Results}

Concentration and time effects of Angelica Sinensis on the viability of myotubes

The viability of cells in the group without AS treatment was expressed as $100 \%$. As shown in Table 1 , at $24 \mathrm{~h}$, the cell viability of myotubes decreased by $9 \%, 16 \%$, and $26 \%$ when exposed to $10^{4}, 10^{5}$, and $10^{6} \mathrm{ng} / \mathrm{mL}$ of AS, respectively, compared with the cells in the untreated control group. At $48 \mathrm{~h}$, the cell viability of the myotubes decreased by $9 \%, 25 \%$, and $31 \%$ when exposed to $10^{4}$, $10^{5}$, and $10^{6} \mathrm{ng} / \mathrm{mL}$ of AS, respectively, compared with 
Table 1 Effect of Angelica Sinensis on viability of myotubes (\%)

\begin{tabular}{lcccccccc}
\hline & & \multicolumn{6}{c}{ Concentration of Angelica Sinensis treatment (ng/mL) } \\
\cline { 2 - 8 } & Control & $\mathbf{1}$ & $\mathbf{1 0}$ & $\mathbf{1 0}^{\mathbf{2}}$ & $\mathbf{1 0}^{\mathbf{3}}$ & $\mathbf{1 0}^{\mathbf{4}}$ & $\mathbf{1 0}^{\mathbf{5}}$ & $\mathbf{1 0}^{\mathbf{6}}$ \\
\hline $\mathbf{2 4} \mathbf{~ h}$ & $100.00 \pm 3.53$ & $99.45 \pm 4.09$ & $99.89 \pm 5.64$ & $98.18 \pm 4.52$ & $93.94 \pm 6.59$ & $91.24 \pm 4.34$ & $83.62 \pm 5.07^{*}$ & $74.41 \pm 5.88^{*}$ \\
$\mathbf{4 8 ~ h}$ & $100.00 \pm 3.42$ & $100.15 \pm 5.34$ & $100.04 \pm 5.56$ & $100.13 \pm 5.75$ & $93.49 \pm 4.44$ & $91.22 \pm 4.09$ & $75.08 \pm 5.15^{*}$ & $68.82 \pm 4.65^{*}$ \\
$\mathbf{7 2} \mathbf{~ h}$ & $100.00 \pm 3.75$ & $98.56 \pm 3.49$ & $99.42 \pm 5.21$ & $99.17 \pm 4.86$ & $91.31 \pm 3.92$ & $90.64 \pm 3.59$ & $74.93 \pm 5.26^{*}$ & $68.45 \pm 4.53^{*}$ \\
\hline
\end{tabular}

Values are mean $\pm S D(n=4)$. Data were analyzed with one-way ANOVA with dose factors. *Significantly different compared with the control at the same time of culture (Scheffe's post hoc analysis, $P<0.05$ )

the cells in the untreated control group. At $72 \mathrm{~h}$, the cell viability of the myotubes decreased by $9 \%, 25 \%$, and $32 \%$ when exposed to $10^{4}, 10^{5}$, and $10^{6} \mathrm{ng} / \mathrm{mL}$ of $\mathrm{AS}$, respectively, compared with the cells in the untreated control group. The cell viability at concentrations of $10^{5}$ and $10^{6} \mathrm{ng} / \mathrm{mL}$ of AS was significantly decreased compared with the control group after the same period of culturing $(P<0.05)$. The results indicated that AS was not harmful to myotubes at concentrations of 1,10 , or $10^{2} \mathrm{ng} / \mathrm{mL}$. Therefore, an AS concentration of $10 \mathrm{ng} / \mathrm{mL}$ was used to induce hypertrophy in the experiment.

\section{Myotube hypertrophy induced by Angelica Sinensis}

To determine whether AS is functionally critical for myotube hypertrophy, the influence of AS $(10 \mathrm{ng} / \mathrm{mL})$ on myotube thickness was examined. After $72 \mathrm{~h}$ of incubation, highly thickened myotubes were observed in the AS-treated group. The myotube diameter of 2 groups
(NON and AS) exhibited normal distribution. The result indicated that the average myotube diameter in the AS group increased $1.34 \pm 0.13$ fold compared with the NON group $(P<0.05$, Figure 1$)$. This clearly revealed that AS induced myotube hypertrophy.

\section{Involvement of the PI3K/Akt/mTOR pathway in Angelica Sinensis-induced myotube hypertrophy}

To examine the role of the PI3K/Akt/mTOR signaling pathway in AS-induced myotube hypertrophy, pharmacologic experiments were conducted using inhibitors that interfered with this pathway. IGF-1 stimulation that activated the pathway was used as a positive control. The PI3K inhibitor, wortmannin, reduced the diameters of AS-treated myotubes by $25 \%(P<0.05$, Figure $2 \mathrm{~A}, \mathrm{AS})$, and the diameters of the positive controls by approximately $30 \%(P<0.05$, Figure $2 \mathrm{~A}$, IGF-1). The mTOR inhibitor, rapamycin, behaved similarly to wortmannin
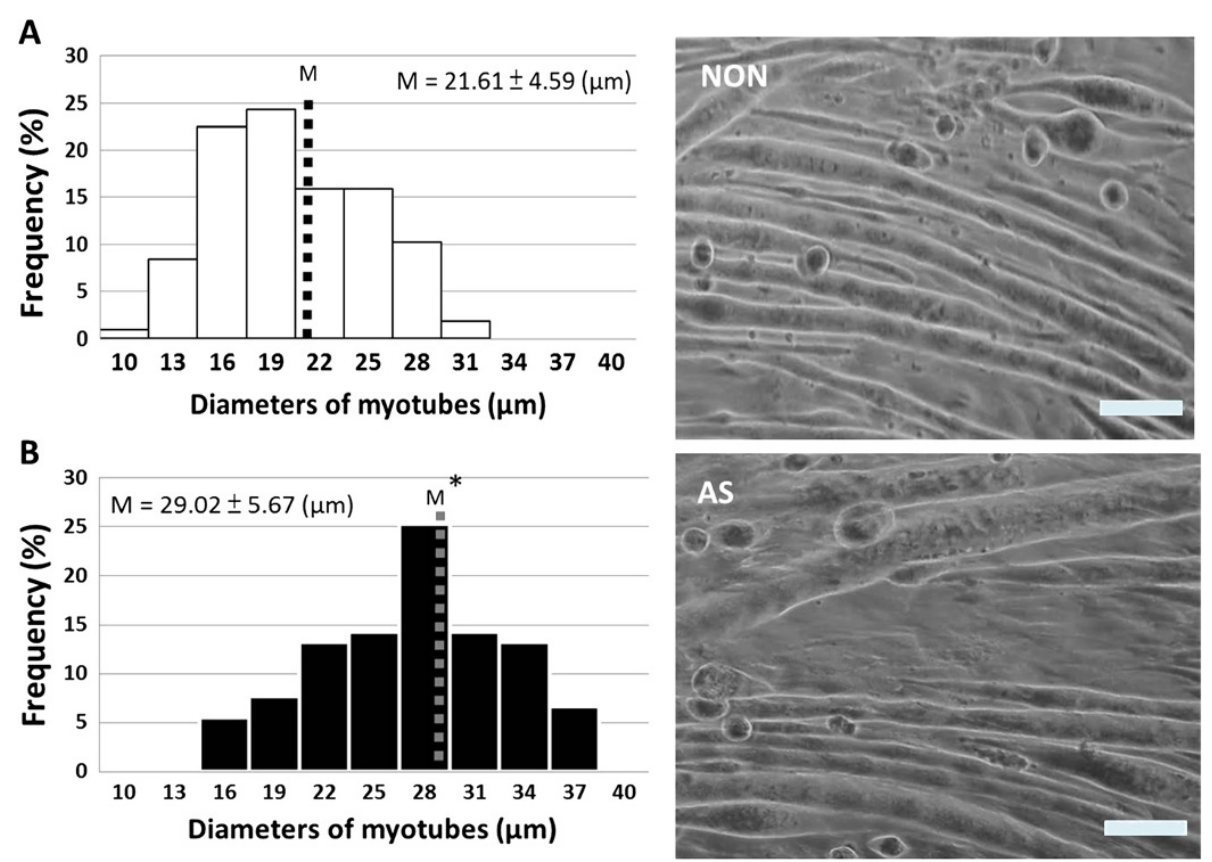

Figure 1 Angelica Sinensis (AS) induced myotube hypertrophy after $\mathbf{7 2} \mathrm{h}$ treatment. Histograms of myotube diameters derived from microscope images. Right panel shows a representative myotubes of image. Scale bar $=50 \mu \mathrm{m}$. Quantification of mean myotube diameter (M) \pm SD. (A) NON, non- Angelica Sinensis supplements, Dulbecco's modified Eagle's medium (DMEM) containing 2\% horse serum (HS), $n=107 ;$ (B) AS, $10 \mathrm{ng} / \mathrm{mL}$ of AS in 2\% HS/DMEM, $\mathrm{n}=91$; n-value represented the myotube numbers from image. The mean of myotube diameters (M) was significantly enlarged in AS group. Data were analyzed with the Student's $t$-test. Statistical significance: ${ }^{*} P<0.05$ vs. NON. 


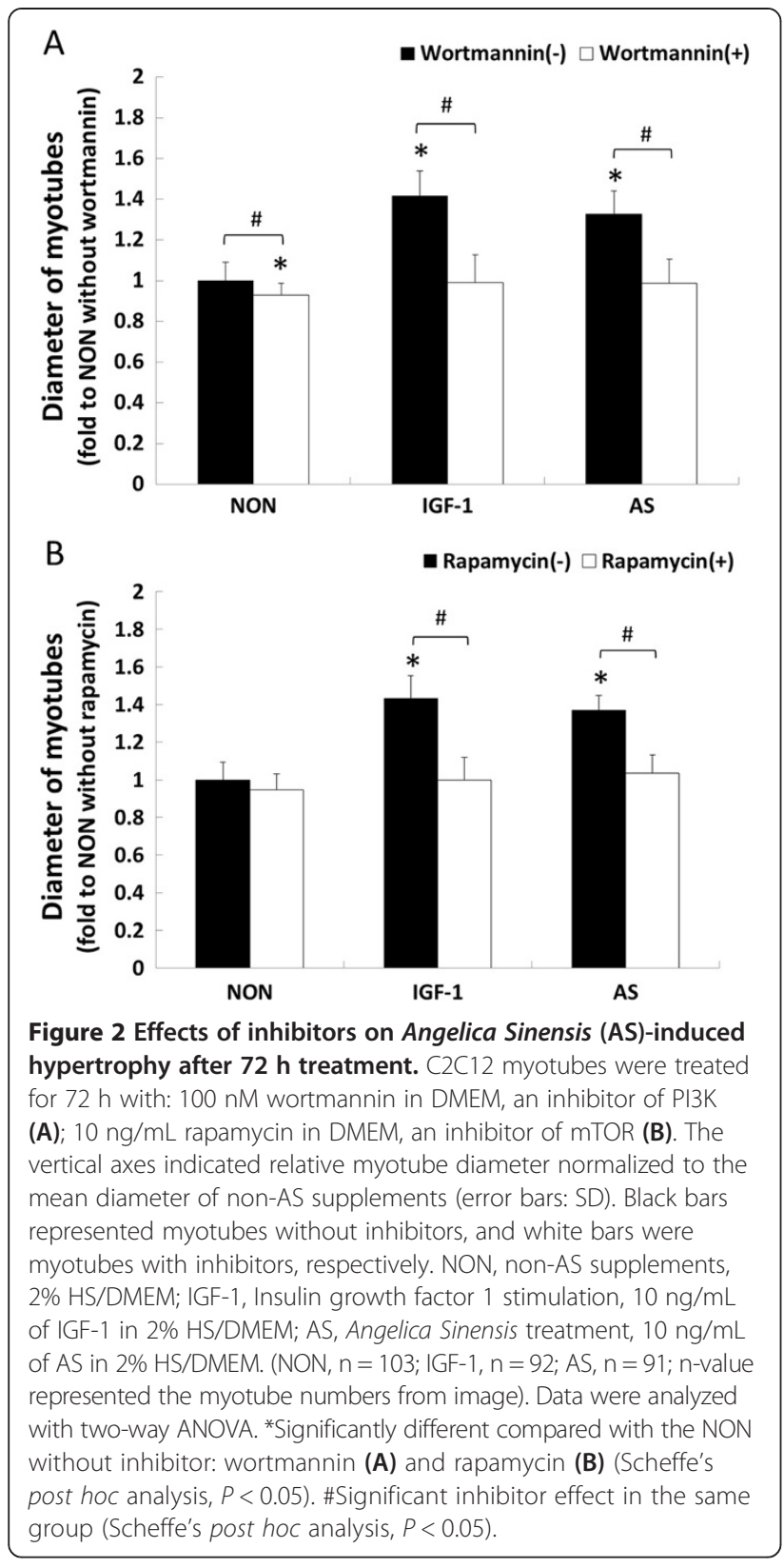

(Figure 2B). These results indicated that the PI3K/Akt/ mTOR pathway played a crucial role in AS-induced myotube hypertrophy.

\section{Akt phosphorylation induced by Angelica Sinensis}

Following the aforementioned indication of the role of the PI3K/Akt/mTOR pathway in AS-induced myotube hypertrophy, we investigated whether Akt phosphorylation was promoted by AS. First, a time-course analysis was performed using western blotting, which showed that 15 and $45 \mathrm{~min}$ of AS treatment significantly elevated the Akt phosphorylation level $(P<0.05)$, as did IGF-1 stimulation regarding the positive control $(P<0.05$, Figure $3 \mathrm{~A})$.
Second, further investigation showed that Akt phosphorylation induced by $15 \mathrm{~min}$ of AS treatment was significantly reduced beyond the non-AS supplement's level $(P<0.05$, Figure $3 \mathrm{~B}$ ), using wortmannin; essentially the same results were obtained in the samples regarding Akt phosphorylation induced by $45 \mathrm{~min}$ of AS treatment (Figure 3C). These data suggested that AS promoted Akt phosphorylation through the PI3K pathway, which was observed in the case of IGF-1 stimulation.

\section{Mamallian target of rapamycin phosphorylation induced by Angelica Sinensis}

The procedure for this experiment resembled the aforementioned time-course analysis. Results showed that 30 min of AS treatment significantly elevated the mTOR phosphorylation level $(P<0.05)$, as did IGF-1 stimulation in the positive control group $(P<0.05$, Figure $4 \mathrm{~A})$. However, a decrease was observed in the phosphorylation level of mTOR between 5 and 30 min after the AS treatment of the myotubes. The mTOR phosphorylation behaved similarly to Akt phosphorylation, but more powerfully expressed the hypertrophy signal in the AS-treated sample. Furthermore, the elevated phosphorylation induced using AS treatment for 30 min was significantly reduced using wortmannin compared with the non-AS supplemented group $(P<0.05$, Figure $4 \mathrm{~B})$. The non-AS supplemented group exhibited a similar reaction. Additionally, the wortmannin inhibition of phosphorylation levels was not significantly different between the 2 groups. As shown in Figures $3 \mathrm{~B}$ and $4 \mathrm{~B}$, the AS-induced hypertrophy through the PI3K/Akt/mTOR phosphorylation pathway was completely inhibited using wortmannin; however, it was unclear whether the hypertrophy was solely induced by the PI3K/Akt/mTOR pathway. Nonetheless, after wortmannin inhibition the AS-induced phosphorylation was significantly reduced. Therefore, PI3K undoubtedly played a major role in hypertrophy.

\section{Discussion}

The primary finding of the present study was that AS increased myotube hypertrophy through the PI3K/Akt/ mTOR pathway. According to a thorough review of relevant research, this is the first study to demonstrate that myotube hypertrophy induced by AS treatment occurs through the PI3K/Akt/mTOR pathway, as does IGF-1induced hypertrophy. Furthermore, treatment with AS increases the activation of the PI3K/Akt/mTOR pathway.

The PI3K/Akt/mTOR pathway was investigated to understand the mechanism through which AS promotes hypertrophy. Activating this pathway promotes skeletal muscle hypertrophy and prevents muscle atrophy [25] because the kinase activity of Akt is essential for IGF-1induced hypertrophy [13]. Akt is a serine-threonine protein kinase that can induce protein synthesis and block 


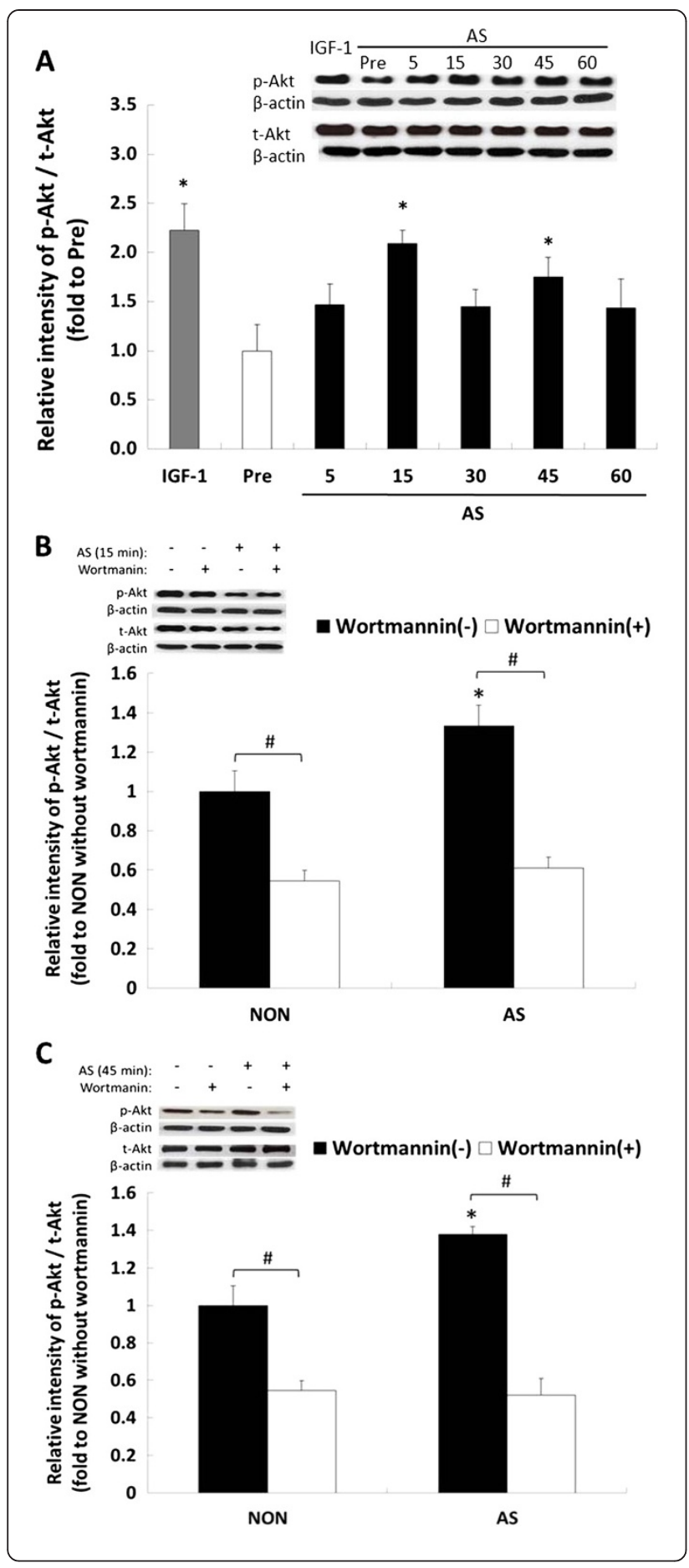

Figure 3 Phosphorylation of Akt induced by Angelica Sinensis (AS). (A) Upper panel showed a representative result of western blot analysis of total- Akt (t-Akt) and phosphor-Akt ( $p$-Akt) levels in the myotubes treated with $10 \mathrm{ng} / \mathrm{mL}$ of IGF-1 in 2\% HS/DMEM for $45 \mathrm{~min}$, or AS (10 ng/mL of AS in 2\% HS/DMEM) for 5 to $60 \mathrm{~min}$.

(B) Akt phosphorylation level at $15 \mathrm{~min}$ in wortmannin-treated myotubes. (C) Akt phosphorylation level at 45 min in wortmannintreated myotubes. $\mathrm{p}$-Akt and t-Akt were normalized by individual $\beta$-actin. The results of the densitometric analysis of the western blot membranes [upper panels in (A), (B) and (C)] were depicted in the lower panels as the ratio of $\mathrm{p}$-Akt against the t-Akt signal (mean \pm $S D, n=3$ ), respectively. Vertical axis represented relative $p$-Akt level compared with pre-treated myotubes (A), or non-treated myotubes (B) and (C). Data were analyzed with one-way ANOVA with time factors in (A). Data were analyzed with two-way ANOVA with group and inhibitor treat as factors in (B) and (C). *Significant time effect compared with pre-treat in (A) (Scheffe's post hoc analysis, $P<0.05$ ). *Significantly different compared with the NON without inhibitor wortmannin in (B) and (C) (Scheffe's post hoc analysis, $P<0.05$ ). \#Significant inhibitor effect in the same group (Scheffe's post hoc analysis, $P<0.05)$.

the transcriptional upregulation of key mediators of skeletal muscle atrophy [26]. No previous studies that examined AS have investigated the regulation of the PI3K/ Akt/mTOR pathway in myotube hypertrophy. Immunoblotting by using antibodies against activated or total Akt and mTOR revealed that AS activated this pathway in myotubes, which clarified AS's hypertrophic effects on myotubes.

Wortmannin, a specific inhibitor of PI3K, was used to distinguish whether AS activated Akt through the classical PI3K pathway or through an alternative pathway. Wortmannin attenuated Akt activation was induced using AS, which demonstrated that Akt activation by using AS is dependent on the PI3K pathway. In this study, we observed that wortmannin inhibited hypertrophy that was promoted using AS, confirming that PI3K-mediated Akt activation by using AS was necessary to induce hypertrophy in myotubes.

Rapamycin is a pharmacologic agent that binds to mTOR and inhibits its functioning [27]. In vitro, when applied to myotube cultures, rapamycin blocks activation of p70S6K downstream of either activated Akt or IGF-1 stimulation $[27,28]$. In this study, we observed that rapamycin inhibited the hypertrophy promoted using AS, which confirmed that Akt-mediated mTOR activation by using AS is necessary to induce hypertrophy in myotubes.

As shown in Figure 4, we observed that myotubes treated with AS for $15 \mathrm{~min}$ or longer had significantly increased levels of PI3K-mediated Akt activation on $\operatorname{Ser}^{473}(P<0.05)$ resulting in hypertrophy, and that activating Akt and its resulting downstream effects was triggered by treatment with AS. AS is responsible for the increase in the activation of mTOR phosphorylation at $\mathrm{Ser}^{2448}$ observed $30 \mathrm{~min}$ after AS treatment (Figure 4A). 


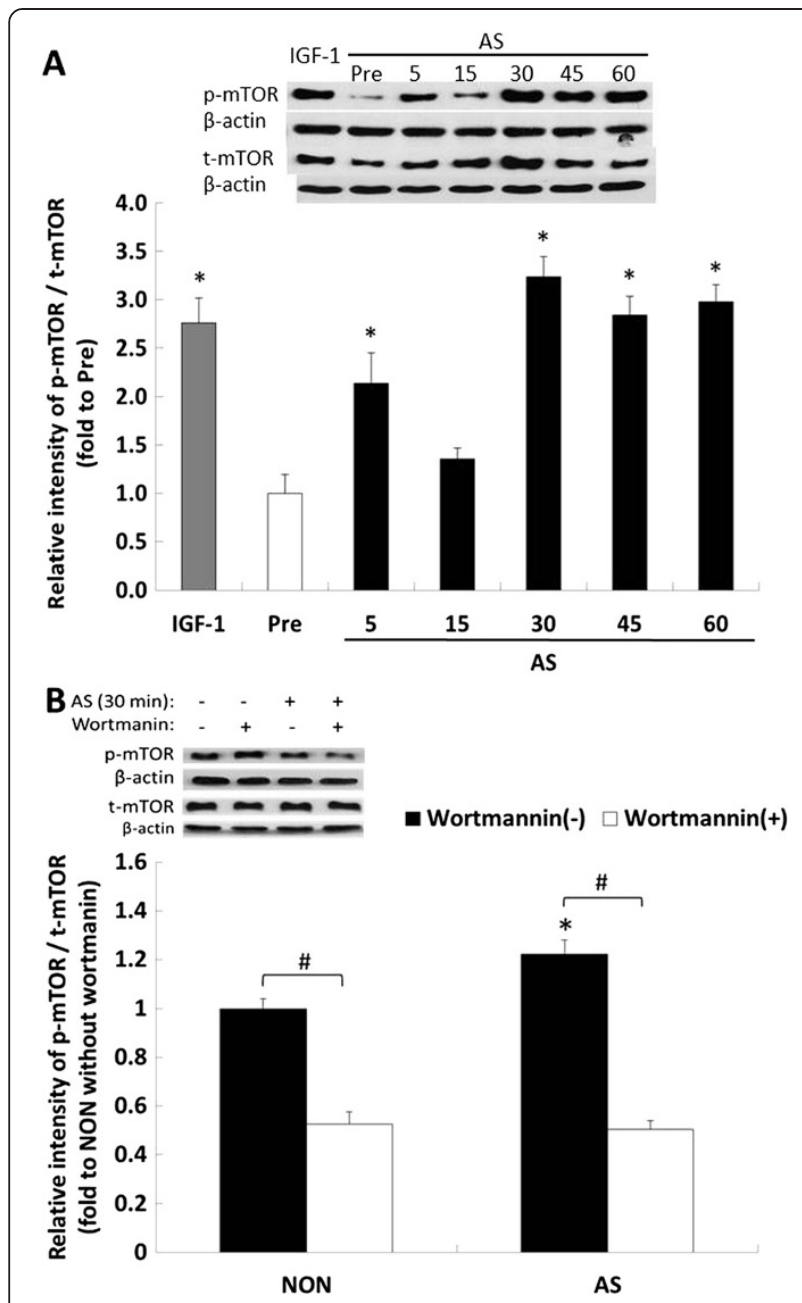

Figure 4 Phosphorylation of mTOR induced by Angelica Sinensis (AS). (A) Upper panel showed a representative result of western blot analysis of total (t-mTOR) and phosphor-mTOR ( $p$-mTOR) levels in the myotubes treated with $10 \mathrm{ng} / \mathrm{mL}$ of IGF-1 in 2\% HS/DMEM for $45 \mathrm{~min}$, or AS (10 ng/mL of AS in 2\% HS/DMEM) for 5 to $60 \mathrm{~min}$. (B) mTOR phosphorylation level at $30 \mathrm{~min}$ in wortmannin-treated myotubes. p-mTOR and t-mTOR were normalized by individual $\beta$-actin. The results of the densitometric analysis of the western blot membranes [upper panels in (A) and (B)] were depicted in the lower panels as the ratio of $p$-mTOR against the t-mTOR signal (mean $\pm S D, n=3$ ), respectively. Vertical axis represented relative $p$-mTOR level compared with pre-treated myotubes (A), or non-treated myotubes (B). Data were analyzed with one-way ANOVA with time factors in (A). Data were analyzed with two-way ANOVA with group and inhibitor treat as factors in (B). * Significant time effect compared with pre-treat in (A) (Scheffe's post hoc analysis, $P<0.05)$. *Significantly different compared with the NON without inhibitor wortmannin in (B) (Scheffe's post hoc analysis, $P<0.05$ ). \#Significant inhibitor effect in the same group (Scheffe's post hoc analysis, $P<0.05$ ).

Akt is a serine-threonine kinase involved in the regulation of cellular metabolism and has been shown to induce rapid skeletal muscle hypertrophy in vivo [29]. Phosphorylation of $\operatorname{Ser}^{473}$ is required for maximal activation of Akt and it appears that Akt might have a relatively short activation period after nutritional stimulation is activated by protein growth factors [30-32]. In this study, the protein level of Akt phosphorylation was observed as early as 5 min after AS treatment and reached maximum protein expression at $15 \mathrm{~min}$. These results were consistent with previous reports [30].

This study revealed that AS increased myotube diameter and seemed to be mediated via the mTOR pathway. Because $2 \%$ horse serum was used in all treatment media throughout the study, the mechanism might have resulted from the direct effect of AS on the mTOR pathway or the enhanced mTOR pathway caused by facilitation of the binding of IGF-1 to its receptor. However, our results revealed that myotube diameter in the AS group was significantly thickened compared with that of the NON group, but not the IGF-1 group (Figure 2). According to our in vitro data, even if horse serum contained IGF-1, AS-induced myotube hypertrophy did not entirely enhance the mTOR pathway by facilitating the binding of IGF-1 to its receptor (Figure 2). We suggest that further study by using a serum free medium is required to investigate how AS activates the PI3K/Akt/ mTOR pathway.

mTOR is a $289 \mathrm{kDa}$ serine-threonine kinase partially downstream of Akt and is responsible for the complex integration of anabolic stimuli mediating cell growth [32]. Although AKT phosphorylated mTOR at $2 \mathrm{COOH}-$ terminal sites ( $\mathrm{Thr}^{2446}$ and $\mathrm{Ser}^{2448}$ ) in vitro, $\mathrm{Ser}^{2448}$ was the major phosphorylation site in insulin-stimulated or -activated AKT-phosphorylating human skeletal muscle cells [32,33]. Phosphorylation of mTOR at Ser ${ }^{2448}$ has become a popular biomarker for the activation state of skeletal muscle hypertrophy signaling pathways and the activation status of mTOR [28,30,34]. A previous study reported that $\mathrm{mTOR}$ is a direct substrate for the Akt kinase and identified $\operatorname{Ser}^{2448}$ as the Akt target site in mTOR [35]. In addition to the regulation of mTOR by using the PI3K/Akt pathway, others have provided evidence that the $\operatorname{Ser}^{2448}$ phosphorylation primarily reflects a feedback signal to mTOR from its downstream target, p70S6 kinase (S6K1) [36]. Figure 4 shows that $30 \mathrm{~min}$ of AS treatment significantly elevated the mTOR phosphorylation level at $\operatorname{Ser}^{2448}$. The negative regulation of skeletal muscle hypertrophy through the p70S6 pathway was a possible reason for the increased phosphorylated mTOR at the $\operatorname{Ser}^{2448}$ site between 30 and $60 \mathrm{~min}$ that was observed. However, downstream signaling factors were required to sustain $\mathrm{AKT} / \mathrm{mTOR}$ signaling. Our results suggested that, at least regarding the cell types examined in this study, $\mathrm{Ser}^{2448}$ phosphorylation exhibited both direct and indirect reactions by using AS stimulation and markers of Akt activation. 


\section{Conclusion}

The results confirm that AS induces hypertrophy in myotubes through the PI3K/Akt/mTOR pathway.

\section{Additional file}

Additional file 1: Figure S1. The chromatogram of ferulic acid (FA) in Angelica Sinensis. Figure S2. Various concentration of Angelica Sinensis (AS) induced myotube hypertrophy after $72 \mathrm{~h}$ treatment. Scale bar $=50 \mu \mathrm{m}$.

\section{Competing interests}

The authors declare that they have no competing interests.

\section{Authors' contributions}

TSY designed the experiments, performed the laboratory experiments, analyzed the data, interpreted the results, prepared figures, and wrote the manuscript. $\mathrm{CCH}$ and SCY edited and revised manuscript. MCH and JFL supervised the study design and revised the manuscript. All authors discussed the results and implications and commented on the manuscript at all stages. All authors read and approved the final manuscript.

\section{Acknowledgment}

We thank Brion Research Institute in Taiwan for the assistance in ferulic acid determination. This study was supported by the National Science Council, Taiwan, grants NSC 99-2410-H-179-006-MY2.

\section{Author details}

${ }^{1}$ School of Nutrition and Health Sciences, Taipei Medical University, Taipei 11031, Taiwan. ${ }^{2}$ Department of Anatomy, School of Medicine, Taipei Medical University, Taipei 11031, Taiwan. ${ }^{3}$ Department of Sports Medicine, Kaohsiung Medical University, Kaohsiung 80708, Taiwan. ${ }^{4}$ Graduate Institute of Sports Science, National Taiwan Sport University, Taoyuan 33301, Taiwan. ${ }^{5}$ Department of Nutrition and Health Sciences, Chang Gung University of Science and Technology, Taoyuan 33303, Taiwan. ${ }^{6}$ Research Center for Industry of Human Ecology, Chang Gung University of Science and Technology, Taoyuan 33303, Taiwan.

Received: 29 September 2013 Accepted: 25 March 2014 Published: 3 May 2014

\section{References}

1. Fiatarone MA, Marks EC, Ryan ND, Meredith CN, Lipsitz LA, Evans WJ: Highintensity strength training in nonagenarians. Effects on skeletal muscle. JAMA 1990, 263(22):3029-3034.

2. Rantanen $T$, Avlund $K$, Suominen $H$, Schroll M, Frändin $K$, Pertti E: Muscle strength as a predictor of onset of $A D L$ dependence in people aged 75 years. Aging Clin Exp Res 2002, 14(suppl 3):10-15.

3. Wolfson L, Judge J, Whipple R, King M: Strength is a major factor in balance, gait, and the occurrence of falls. J Gerontol A Biol Sci Med Sci 1995, 50:64-67.

4. Frost RA, Lang $\mathrm{CH}$ : Protein kinase B/Akt: a nexus of growth factor and cytokine signaling in determining muscle mass. J Appl Physiol 2007, 103(1):378-387.

5. Glass DJ: Skeletal muscle hypertrophy and atrophy signaling pathways. Int J Biochem Cell Biol 2005, 37(10):1974-1984.

6. Schiaffino S, Mammucari C: Regulation of skeletal muscle growth by the IGF1-Akt/PKB pathway: insights from genetic models. Skelet Muscle 2011 $1(1): 1-14$.

7. Hulmi JJ, Tannerstedt J, Selanne H, Kainulainen H, Kovanen V, Mero AA: Resistance exercise with whey protein ingestion affects mTOR signaling pathway and myostatin in men. J App/ Physio/ 2009, 106(5):1720-1729.

8. Kumar V, Selby A, Rankin D, Patel R, Atherton P, Hildebrandt W, Williams J, Smith K, Seynnes O, Hiscock N, Rennie MJ: Age-related differences in the dose-response relationship of muscle protein synthesis to resistance exercise in young and old men. J Physiol 2009, 587(Pt 1):211-217.

9. Morissette MR, Cook SA, Buranasombati C, Rosenberg MA, Rosenzweig A Myostatin inhibits IGF-l-induced myotube hypertrophy through Akt. Am J Physiol Cell Physiol 2009, 297(5):C1124-C1132.
10. Sandri M, Sandri C, Gilbert A, Skurk C, Calabria E, Picard A, Walsh K, Schiaffino S, Lecker SH, Goldberg AL: Foxo transcription factors induce the atrophy-related ubiquitin ligase atrogin-1 and cause skeletal muscle atrophy. Cell 2004, 117(3):399-412.

11. Stitt TN, Drujan D, Clarke BA, Panaro F, Timofeyva Y, Kline WO, Gonzalez M Yancopoulos GD, Glass DJ: The IGF-1/PI3K/Akt pathway prevents expression of muscle atrophy-induced ubiquitin ligases by inhibiting FOXO transcription factors. Mol Cell 2004, 14(3):395-403.

12. Bodine SC, Stitt TN, Gonzalez M, Kline WO, Stover GL, Bauerlein R, Zlotchenko E, Scrimgeour A, Lawrence JC, Glass DJ, Yancopoulos GD: Akt/ mTOR pathway is a crucial regulator of skeletal muscle hypertrophy and can prevent muscle atrophy in vivo. Nat Cell Biol 2001, 3(11):1014-1019.

13. Rommel C, Bodine SC, Clarke BA, Rossman R, Nunez L, Stitt TN, Yancopoulos GD, Glass DJ: Mediation of IGF-1-induced skeletal myotube hypertrophy by $\mathrm{PI}(3) \mathrm{K} / \mathrm{Akt} / \mathrm{mTOR}$ and PI(3)K/Akt/GSK3 pathways. Nat Cell Biol 2001, 3(11):1009-1013

14. Yaffe D, Saxel O: Serial passaging and differentiation of myogenic cells isolated from dystrophic mouse muscle. Nature 1977, 270(5639):725-727.

15. Hitachi K, Nakatani M, Tsuchida K: Myostatin signaling regulates Akt activity via the regulation of miR-486 expression. Int J Biochem Cell Biol 2013, 47C:93-103.

16. Latres $E$, Amini AR, Amini AA, Griffiths J, Martin FJ, Wei Y, Lin HC, Yancopoulos GD, Glass DJ: Insulin-like growth factor-1 (IGF-1) inversely regulates atrophy-induced genes via the phosphatidylinositol 3-kinase/ Akt/mammalian target of rapamycin (PI3K/Akt/mTOR) pathway. J Biol Chem 2005, 280(4):2737-2744.

17. Zhao KJ, Dong TT, Tu PF, Song ZH, Lo CK, Tsim KW: Molecular genetic and chemical assessment of radix Angelica (Danggui) in China. J Agric Food Chem 2003, 51(9):2576-2583.

18. Song ZY: The Modern Studies on the Chinese Meteria Medica. Volume 2 Beijing, China: Peking Union Medical College and Beijing Medical University Press; 1996:1-25.

19. Wagner H, Bauer R, Xiao PG, Chen JM, Michler H: Chinese Drug Monographs and Analysis, Angelica sinensis. Volume 3. Wald, Germany: Verlag; 2001

20. Chang CJ, Chiu JH, Tseng LM, Chang CH, Chien TM, Wu CW, Lui WY Modulation of HER2 expression by ferulic acid on human breast cancer MCF7 cells. Eur J Clin Invest 2006, 36(8):588-596.

21. Liu C, Li J, Meng FY, Liang SX, Deng R, Li CK, Pong NH, Lau CP, Cheng SW, Ye JY, Chen JL, Yang ST, Yan H, Chen S, Chong BH, Yang M: Polysaccharides from the root of Angelica sinensis promotes hematopoiesis and thrombopoiesis through the PI3K/AKT pathway. BMC Complement Altern Med 2010, 10:79-90.

22. Lawson MA, Purslow PP: Differentiation of myoblasts in serum-free media: effects of modified media are cell line-specific. Cells Tissues Organs 2000, 167(2-3):130-137.

23. Sasai N, Agata N, Inoue-Miyazu M, Kawakami K, Kobayashi K, Sokabe M, Hayakawa K: Involvement of PI3K/Akt/TOR pathway in stretch-induced hypertrophy of myotubes. Muscle Nerve 2010, 41(1):100-106.

24. Chinese Pharmacopoeia Committee: China medical science and technology in pharmacopoeia of the people's republic of China: chemical drugs and biological agents. Beijing: Chinese Medical Science Press; 2010:124-125.

25. Bamman MM, Shipp JR, Jiang J, Gower BA, Hunter GR, Goodman A, McLafferty CL Jr, Urban RJ: Mechanical load increases muscle IGF-I and androgen receptor mRNA concentrations in humans. Am J Physiol Endocrinol Metab 2001, 280(3):E383-E390.

26. Wilson EM, Rotwein P: Selective control of skeletal muscle differentiation by Akt1. J Biol Chem 2007, 282(8):5106-5110.

27. Willett M, Cowan JL, Vlasak M, Coldwell MJ, Morley SJ: Inhibition of mammalian target of rapamycin (mTOR) signalling in $\mathrm{C} 2 \mathrm{C} 12$ myoblasts prevents myogenic differentiation without affecting the hyperphosphorylation of 4E-BP1. Cell Signal 2009, 21(10):1504-1512.

28. Gran P, Cameron-Smith D: The actions of exogenous leucine on mTOR signalling and amino acid transporters in human myotubes. BMC Physiol 2011, 11:10.

29. Lai KMV, Gonzalez M, Poueymirou WT, Kline WO, Na E, Zlotchenko E, Stitt TN, Economides AN, Yancopoulos GD, Glass DJ: Conditional activation of Akt in adult skeletal muscle induces rapid hypertrophy. Mol Cell Biol 2004, 24(21):9295-9304

30. Basualto-Alarcón C, Jorquera G, Altamirano F, Jaimovich E, Estrada M: Testosterone signals through mTOR and androgen receptor to induce muscle hypertrophy. Med Sci Sports Exerc 2013, 45(9):1712-1720. 
31. Blomstrand E, Eliasson J, Karlsson HK, Kohnke R: Branched-chain amino acids activate key enzymes in protein synthesis after physical exercise. J Nutr 2006, 136(1):269S-273S.

32. Farnfield MM, Breen L, Carey KA, Garnham A, Cameron-Smith D: Activation of mTOR signalling in young and old human skeletal muscle in response to combined resistance exercise and whey protein ingestion. Appl Physiol Nutr Metab 2012, 37(1):21-30.

33. Kakigi R, Yoshihara T, Ozaki H, Ogura Y, Ichinoseki-Sekine N, Kobayashi H, Naito $\mathrm{H}$ : Whey protein intake after resistance exercise activates mTOR signaling in a dose-dependent manner in human skeletal muscle. Eur $\lrcorner$ Appl Physiol. in press.

34. Trendelenburg AU, Meyer A, Rohner D, Boyle J, Hatakeyama S, Glass DJ: Myostatin reduces Akt/TORC1/p70S6K signaling, inhibiting myoblast differentiation and myotube size. Am J Physiol Cell Physiol 2009, 296(6):C1258-C1270.

35. Dennis PB, Jaeschke A, Saitoh M, Fowler B, Kozma SC, Thomas G: Mammalian TOR: a homeostatic ATP sensor. Science 2001, 294(5544):1102-1105.

36. Chiang GG, Abraham RT: Phosphorylation of mammalian target of rapamycin (mTOR) at Ser-2448 is mediated by p70S6 kinase. J Biol Chem 2005, 280(27):25485-25490.

doi:10.1186/1472-6882-14-144

Cite this article as: Yeh et al: Angelica Sinensis promotes myotube hypertrophy through the PI3K/Akt/mTOR pathway. BMC Complementary and Alternative Medicine 2014 14:144.

\section{Submit your next manuscript to BioMed Central and take full advantage of:}

- Convenient online submission

- Thorough peer review

- No space constraints or color figure charges

- Immediate publication on acceptance

- Inclusion in PubMed, CAS, Scopus and Google Scholar

- Research which is freely available for redistribution 